\title{
Variation of the character of spin-orbit interaction by Pt intercalation underneath graphene on $\operatorname{Ir}(111)$
}

\author{
I. I. Klimovskikh, ${ }^{1}$ O. Vilkov, ${ }^{1}$ D. Yu. Usachov,${ }^{1}$ A. G. Rybkin, ${ }^{1}$ S. S. Tsirkin,,${ }^{1,2,3}$ M. V. Filianina, ${ }^{1}$ \\ K. Bokai, ${ }^{1}$ E. V. Chulkov, ${ }^{1,2,3,4}$ and A. M. Shikin ${ }^{1}$ \\ ${ }^{1}$ Saint Petersburg State University, Physical Department, Saint Petersburg, 198504, Russia \\ ${ }^{2}$ Donostia International Physics Center (DIPC), 20018 San Sebastián/Donostia, Basque Country, Spain \\ ${ }^{3}$ Tomsk State University, Laboratory for Nanostructured Surfaces and Coatings, 634050 Tomsk, Russia \\ ${ }^{4}$ Departamento de Física de Materiales UPV/EHU, Centro de Física de Materiales CFM-MPC \\ and Centro Mixto CSIC-UPV/EHU, 20080 San Sebastián/Donostia, Basque Country, Spain \\ (Received 7 May 2015; revised manuscript received 20 July 2015; published 1 October 2015)
}

\begin{abstract}
The modification of the graphene spin structure is of interest for novel possibilities of application of graphene in spintronics. The most exciting of them demand not only high value of spin-orbit splitting of the graphene states, but non-Rashba behavior of the splitting and spatial modulation of the spin-orbit interaction. In this work we study the spin and electronic structure of graphene on $\operatorname{Ir}(111)$ with intercalated Pt monolayer. Pt interlayer does not change the $9.3 \times 9.3$ superlattice of graphene, while the spin structure of the Dirac cone becomes modified. It is shown that the Rashba splitting of the $\pi$ state is reduced, while hybridization of the graphene and substrate states leads to a spin-dependent avoided-crossing effect near the Fermi level. Such a variation of spin-orbit interaction combined with the superlattice effects can induce a topological phase in graphene.
\end{abstract}

DOI: 10.1103/PhysRevB.92.165402

PACS number(s): 73.22.Pr, 75.70.Tj

\section{INTRODUCTION}

Spin-dependent effects in nonmagnetic systems became very attractive for researchers in recent years. The most important breakthroughs in this field are Rashba systems [1-4] and topological insulators [5-7]. Both are characterized by two-dimensional electronic states, which are spin polarized owing to the high magnitude of spin-orbit interaction (SOI). The unique spin structure allows one to use these systems in spintronics and quantum computing [8-10].

Another low-dimensional material potentially applicable for spintronics is graphene, which has massless Dirac fermions and large spin relaxation length [11,12]. Although graphene due to a small atomic number of carbon atoms has an extremely low value of intrinsic SOI, it was recently shown, that an interaction with the substrate can induce spin-dependent effects in graphene [13-15]. Hybridization of the graphene and substrate $d$ states leads to the spin splitting of the Dirac conelike $\pi$ state, with the value of splitting up to $100 \mathrm{meV}$. In graphene on $\mathrm{Au} / \mathrm{Ni}(111)$ and on $\operatorname{Ir}(111)$ spin-dependent hybridization takes place in the regions far from the Dirac point and the Fermi level. Thus, the authors of Refs. [13,14] attributed the spin splitting near the Dirac point to the Rashba effect. On the other hand, it has been theoretically predicted that spin-orbit coupling can induce a topological phase in graphene, similar to topological insulators [16]. However, Rashba splitting of the states does not open the gap in the Dirac cone, and is useless for this purpose. The topological gap in graphene can be opened when "intrinsic" SOI is higher than Rashba SOI. The term "intrinsic" SOI means that it can be described by additional terms in Hamiltonian, which is similar to atomic SOI $[16,17]$.

Two mechanisms, which can create the spin-orbit gap in graphene with impurities were proposed by Weeks et al. [17] and $\mathrm{Hu}$ et al. [18]. The first is based on the interaction of graphene with heavy $p$ adatoms. The authors revealed an enhanced "intrinsic" SOI and the formation of a quantum Spin
Hall (QSH) phase in graphene with Tl or In impurities. The second method relies on the hybridization of the graphene states in the region of the Dirac point with the $5 d$ impurity states near the Fermi level. Moreover, it was shown [19] that the intercalation of $5 d$ metals underneath graphene can also induce an appearance of the spin-orbit gap and topological insulator phase. Recently, we had observed similar effects in graphene on $\operatorname{Pt}(111)$ [20]. This system is characterized by a spin-dependent avoided-crossing effect between the $\mathrm{Pt} 5 d$ and graphene $\pi$ states directly at the Fermi level. It has been shown that the spin-orbit gap is opened at the Dirac point and the spin structure near the Dirac point cannot be described by Rashba SOI.

The latest experimental results on a formation of the topological phase are related to graphene on $\operatorname{Ir}(111)$ with intercalated $\mathrm{Pb}$ islands [21]. The authors observed the appearance of quasi-Landau levels without an external magnetic field. Monolayer islands of $\mathrm{Pb}$ induce a strong and spatially modulated SOI in graphene, which acts as a pseudomagnetic field. Variation of the SOI in graphene is performed by a superstructure of $\mathrm{Pb}$ atoms, which generates spin-dependent superlattice potential. At the edges of the regions the SO coupling varies and in-plane spin-polarized topologically protected modes are expected.

Graphene on $\operatorname{Ir}(111)$ is a well-studied system with a weak interaction with the substrate [22-26]. It is characterized by the $9.3 \times 9.3$ moiré superstructure, and geometrical corrugation which arises from the lattice mismatch of graphene with respect to the $\operatorname{Ir}(111)$. Such a superstructure leads to formation in the spectral function of graphene/ $\operatorname{Ir}(111)$ of additional features, replicas, and minigaps [24]. It has been shown, that near the $\bar{K}$ point of the Brillouin zone (BZ) of graphene, in the region of the bulk band gap of $\operatorname{Ir}(111)$, the $\pi$ state is spin split, with the Rashba behavior and the value of the splitting of $50 \mathrm{meV}$ [14].

In this work we analyze the electronic and spin structure of graphene on $\operatorname{Ir}(111)$ with an intercalated monolayer of Pt. Since the Pt $5 d$ states are localized near the Fermi level, their 
(a)

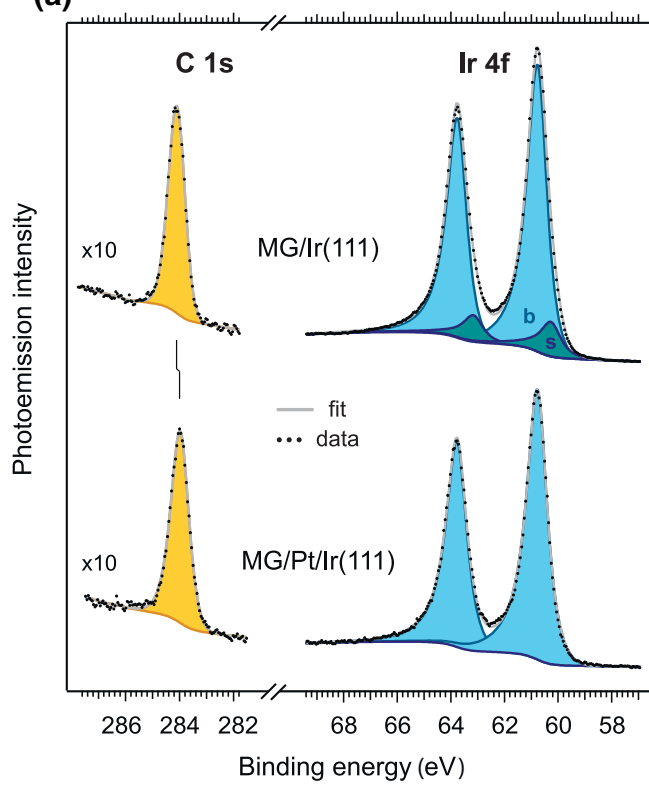

(b)

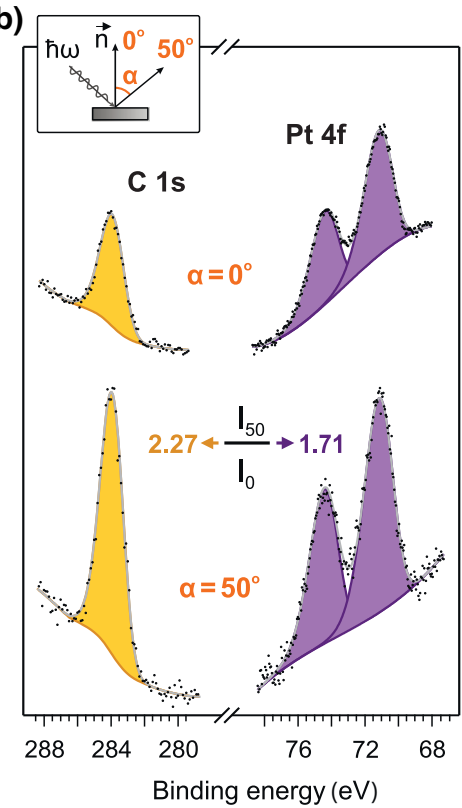

(c)

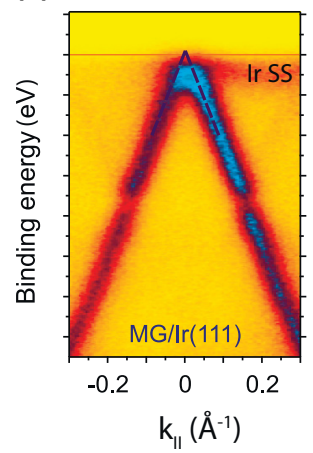

(e)

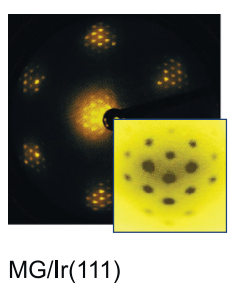

(d)

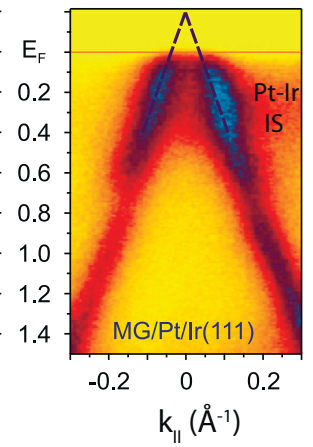

(f)

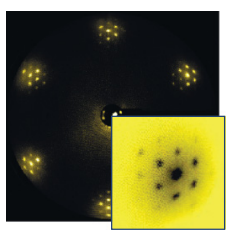

$\mathrm{MG} / \mathrm{Pt} / \mathrm{r}(111)$

FIG. 1. (Color online) (a) Experimental XPS spectra and fitting data of C $1 s$ and $\operatorname{Ir} 4 f$ for MG/Ir(111) (upper) and MG/Pt/Ir(111) (lower), taken at normal emission with the photon energy of $1486.6 \mathrm{eV}$. (b) XPS spectra of $\mathrm{C} 1 \mathrm{~s}$ and $\mathrm{Pt} 4 f$ of MG/Pt/Ir(111) for normal emission (upper) and for $50^{\circ}$ emission angle (lower). (c) ARPES spectra in the direction perpendicular to $\bar{\Gamma} \bar{K}$ for $\mathrm{MG} / \operatorname{Ir}(111)$ and (d) for MG/Pt/Ir(111) using a photon energy of $21.2 \mathrm{eV}$. (e), (f) LEED patterns of MG/Ir(111) and MG/Pt/Ir(111), correspondingly. Insets show zoomed areas around reflexes.

spin-dependent hybridization with the $\pi$ state of graphene is expected near the Dirac point. This effect can lead to a significantly higher SOI variation in graphene, and as a consequence, more effective generation of a topological phase.

\section{EXPERIMENTAL AND COMPUTATIONAL DETAILS}

The experiments were carried out at Helmholtz-Zentrum Berlin (BESSY II) at the UE112-SGM and U125/2-SGM beamlines and in Research Resource Center of Saint Petersburg State University "Physical methods of surface investigation" with a Scienta R4000 energy analyzer. The spin-resolved photoemission spectra were measured using a Mott spin detector operated at $26 \mathrm{keV}$. The total energy resolution during experiments was $50 \mathrm{meV}$. The spin angleresolved photoemission spectroscopy (ARPES) measurements were carried out with the angular resolution of $1^{\circ}$, which corresponds to a momentum resolution of $0.07 \AA^{-1}$ at a photon energy of $62 \mathrm{eV}$. The measurable spin splitting is not limited by the energy resolution, but rather by the acquired statistics. Our estimations of the splitting between the resolved features in photoemission spectra were derived from the fitting of the spectrum features and from the procedures, described in Ref. [14]. The spin asymmetry can be calculated as

$$
A=\frac{N_{L}-N_{R}}{N_{L}+N_{R}},
$$

where $N_{L}$ and $N_{R}$ are the number of electrons counted in the detectors. The background offsets were obtained by the measuring of a reference magnetic sample upon reversal inplane magnetization. The resulting asymmetry yields the spin- resolved intensity spectra

$$
I_{\text {up, down }}=(1 \pm A / S) I_{S} / 2,
$$

where $I_{s}=N_{L}+N_{R}$ and $S$ is a so-called Sherman function.

A clean surface of $\operatorname{Ir}(111)$ was prepared by a well-known procedure, including ion sputtering followed by annealing at $900{ }^{\circ} \mathrm{C}$ in an oxygen atmosphere at a partial pressure of $10^{-7}$ mbar with subsequent flash-annealing up to $1400^{\circ} \mathrm{C}$. The crystalline order and purity of the surface were verified by lowenergy electron diffraction (LEED) and x-ray photoemission spectroscopy (XPS). The graphene monolayer was synthesized on $\operatorname{Ir}(111)$ by cracking of the propylene $\left(\mathrm{C}_{3} \mathrm{H}_{6}\right)$ at a pressure of $2 \times 10^{-7}$ mbar. Intercalation of $\mathrm{Pt}$ was achieved by deposition of a nominal monolayer coverage of $\mathrm{Pt}$ on graphene followed by brief annealing at $700^{\circ} \mathrm{C}$.

Density functional theory (DFT) calculations of the electronic structure and spin polarization of a $\mathrm{Pt}$ monolayer on $\operatorname{Ir}(111)$ were performed using the VASP code [27,28], following the projector-augmented wave (PAW) method $[29,30]$ and generalized gradient approximation (GGA-PBE) [31] for the exchange-correlation energy. The spin-SOI is described within the second variation method [32]. The calculations were performed in a slab geometry: the $\operatorname{Ir}(111)$ substrate is modeled by an 18-layer film, with one side clean and the other covered with a Pt monolayer in the $1 \times 1$ structure.

\section{RESULTS}

For characterization of the crystal structure of the system graphene/Ir(111) with intercalated atoms of Pt LEED and XPS measurements were carried out. Figure 1(a) presents C $1 s$ and $\operatorname{Ir} 4 f$ spectra of graphene on $\operatorname{Ir}(111)$ before (upper 
(a)

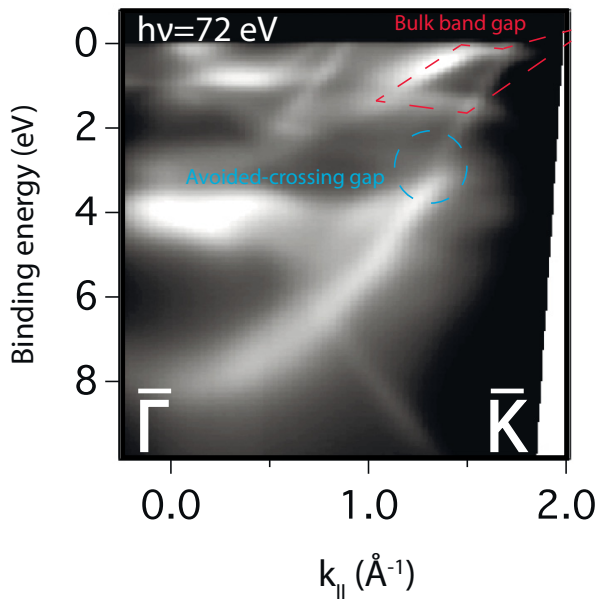

(b)

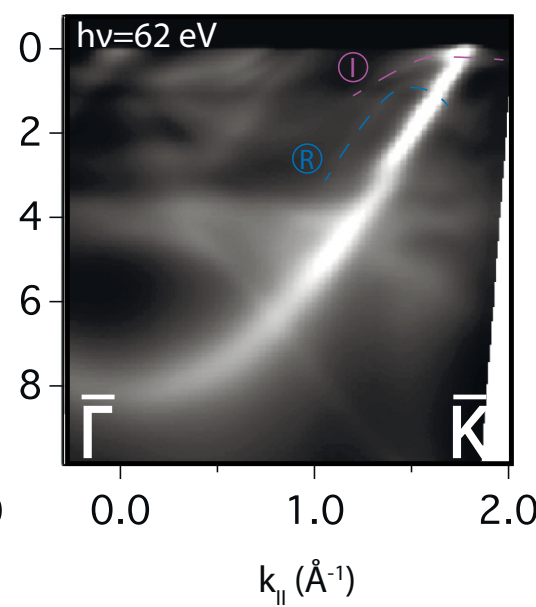

(c)

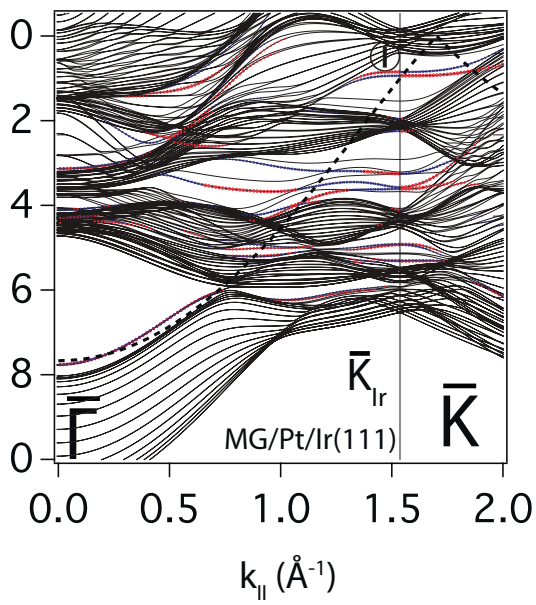

FIG. 2. (Color online) ARPES spectra in the $\bar{\Gamma} \bar{K}$ direction for MG/Pt/Ir(111) with using a photon energy of $62 \mathrm{eV}$ (a) and $72 \mathrm{eV}$ (b). (c) The DFT calculated band structure of $\mathrm{Pt} / \mathrm{Ir}(111)$. The size of the red and blue symbols indicates the weight of an electronic state on the Pt and surface Ir atoms. Red and blue colors correspond to different signs of the projection of spin onto the in-plane perpendicular to the wave vector. The black dashed line represents dispersion of the $\pi$ state of freestanding graphene.

spectra) and after (lower spectra) intercalation of Pt. Data were taken at a photon energy of $1486.6 \mathrm{eV}$. It is seen, that before Pt intercalation, the Ir $4 f$ line consists of two components, derived from bulk (B) and interface layer (S). These features are in agreement with previous studies of the graphene/ $\operatorname{Ir}(111)$ system [22]. After Pt deposition and annealing of the system [lower part in Fig. 1(a)], the interface components of the Ir $4 f$ spectrum disappear, testifying to the formation of a uniform Pt monolayer between the graphene and $\operatorname{Ir}(111)$ substrate. Furthermore, a slight shift of $0.1 \mathrm{eV}$ of $\mathrm{C} 1 s$ peak to lower binding energy (BE) is observed what can be the result of the charge transfer between the graphene monolayer and the $\mathrm{Pt} / \mathrm{Ir}(111)$ substrate.

The intercalation of $\mathrm{Pt}$ atoms underneath graphene can be confirmed by comparison of the relations between the intensities of the $\mathrm{C} 1 s$ and $\mathrm{Pt} 4 f$ peaks in the photoemission spectra taken at various emission angles [see inset in Fig. 1(b)]. The intensity of the $\mathrm{C} 1 s$ line is enhanced at grazing angles much higher compared to the $\mathrm{Pt} 4 f$ spectrum, as shown in Fig. 1(b). Hence, the Pt film is covered with a carbon layer. A detailed quantitative analysis, based on different depths of probing for various emission angles, gives the value of a nearly full monolayer of Pt atoms intercalated underneath graphene.

LEED patterns for pristine graphene on $\operatorname{Ir}(111)$ and after intercalation of the Pt monolayer, taken at $E_{p}=92 \mathrm{eV}$ are presented in Figs. 1(e) and 1(f), correspondingly. As one can see, intercalation of the Pt monolayer does not change the $9.3 \times 9.3$ superstructure, observed for the MG/Ir(111) [24]. It means that intercalated atoms of Pt repeat the substrate lattice, forming a uniform monolayer, with an $\operatorname{Ir}(111)$ unit vector. Thus, the system MG/Pt/Ir(111) can be presented as a Pt monolayer on the $\operatorname{Ir}(111)$ surface with the $1 \times 1$ structure, and graphene on top of $\mathrm{Pt} / \operatorname{Ir}(111)$ with the $9.3 \times 9.3$ superstructure.

Let us turn to an analysis of the electronic structure of the system. It is well known that graphene is characterized by the branches of the $\pi$ states, forming the Dirac cone. The dispersion relations of the states at the $\bar{K}$ point of graphene BZ, in the direction perpendicular to $\bar{\Gamma} \bar{K}$ are shown in Figs. 1(c) and 1(d). Figure 1(c) corresponds to pristine graphene on $\operatorname{Ir}(111)$ and Fig. 1(d) is for graphene after Pt intercalation. The Dirac point is located slightly above $(\sim 0.05 \mathrm{eV})$ the Fermi level for graphene on $\operatorname{Ir}(111)$, which is in accordance with previous works [22-25]. At the binding energy of $0.1 \mathrm{eV}$ an intersection of the graphene $\pi$ state with the Ir surface state takes place. For both systems [Figs. 1(c) and 1(d)] the minigaps induced by crossing with the replicas, caused by moiré superlattice and by periodic corrugation of the system [24], are visible at the $\mathrm{BE}$ of around $0.7 \mathrm{eV}$. After intercalation of a $\mathrm{Pt}$ monolayer underneath graphene [Fig. 1(d)] a quasifreestanding character of the $\pi$ state dispersion is preserved. Meanwhile, the Dirac point shifts approximately to $0.2 \mathrm{eV}$ above the Fermi level. This value is in accordance with the Dirac point position for graphene on $\mathrm{Pt}(111)$ [20]. Moreover, one can see that after intercalation of a Pt monolayer in the region of BE of $0.1-0.3 \mathrm{eV}$ additional states are visible, and local deviations from linear character of $\pi$ state dispersion take place. These features are related to $\operatorname{Ir}(111)$ surface states modified by a Pt monolayer and are better resolved at another geometry of experiment, discussed below.

Figures 2(a) and 2(b) represent experimental dispersion relations of the electronic states of MG/Pt/Ir(111) in the $\bar{\Gamma} \bar{K}$ direction taken at different photon energies. It is seen that at a photon energy of $62 \mathrm{eV}$ [Fig. 2(a)] states of graphene are dominant in the photoemission spectra, due to corresponding matrix elements. With increasing of the photon energy the substraterelated states become more intensive, and at $h v=72 \mathrm{eV}$ [Fig. 2(b)] the $\pi$ state of graphene is seen as a relatively weak feature.

As one can see from Fig. 2(a), electronic structure of the $\mathrm{MG} / \mathrm{Pt} / \mathrm{Ir}(111)$ system is characterized by the pronounced branch of the $\pi$ state of graphene, which has the binding 
(a)

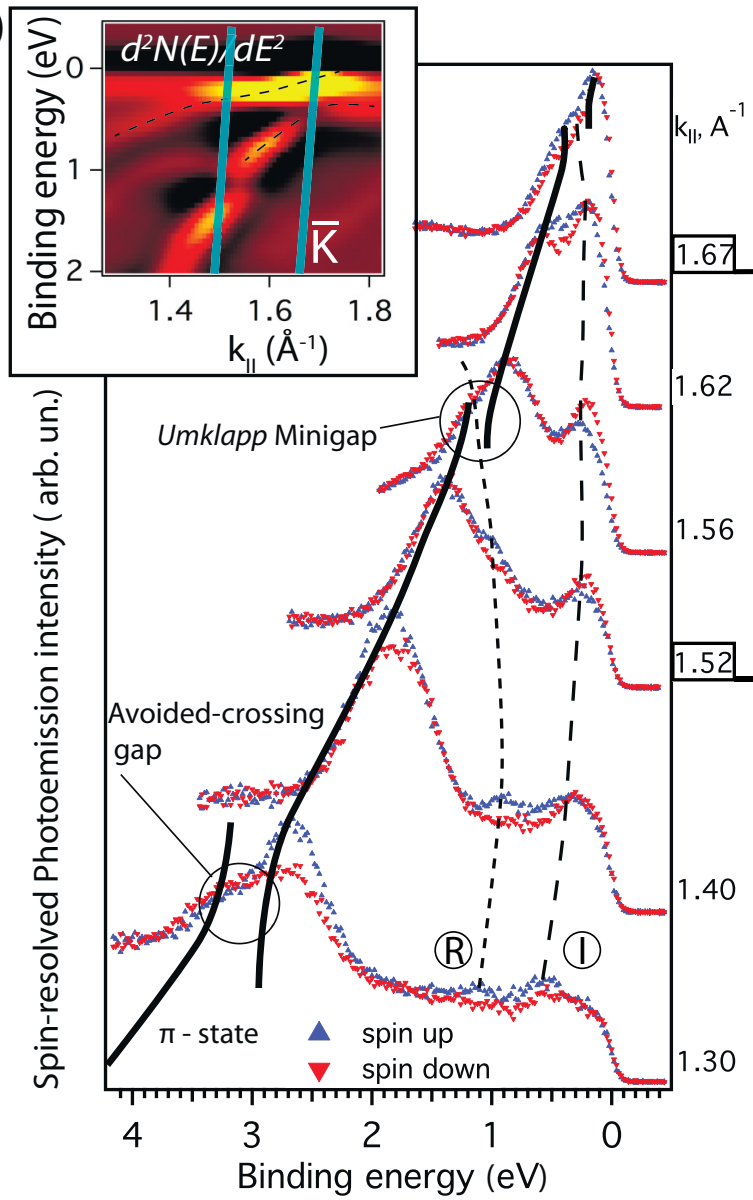

(b)

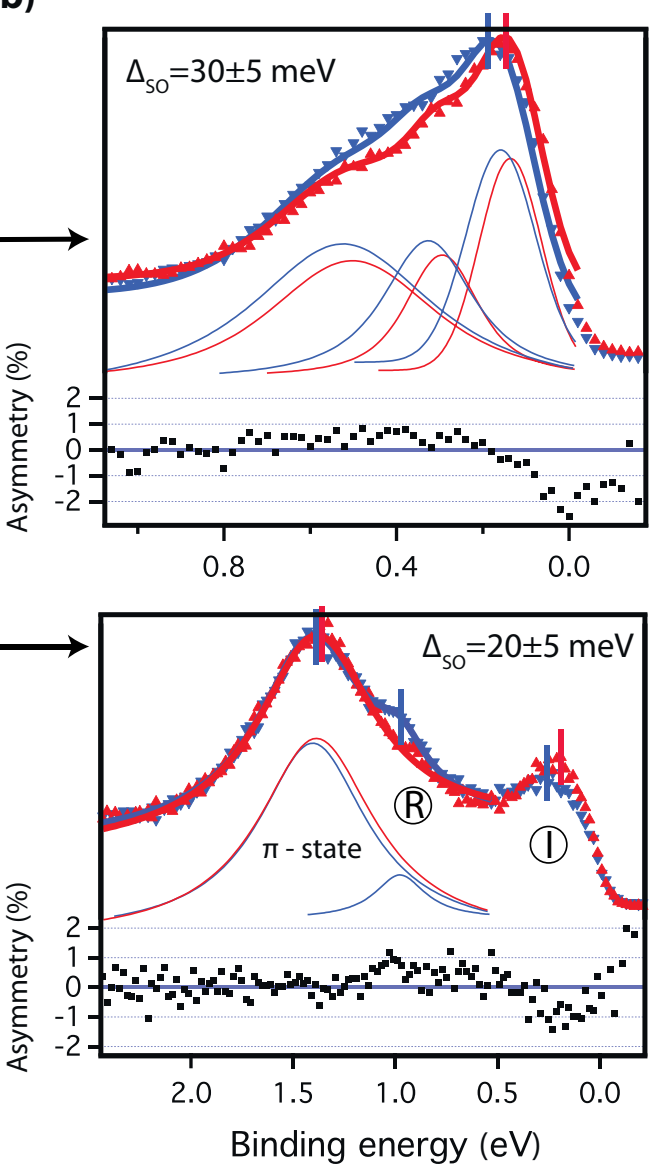

FIG. 3. (Color online) (a) Spin-resolved photoemission spectra for various emission angles in the $\bar{\Gamma} \bar{K}$ direction for MG/Pt/Ir(111). Quantization axis of the spin polarization is perpendicular to the momentum. Second derivative of $N(E)$ from ARPES data in the region of the $\bar{K}$ point of graphene BZ is shown at the inset. Blue lines represent emission angles, for which the (b) spectra were obtained. (b) Fitting of spectra with $k_{\|}=1.52 \AA^{-1}$ and $k_{\|}=1.67 \AA^{-1}$ showing the spin splitting of the $\pi$ state and spin polarization of the replicated band. Raw asymmetry $\left(I_{\text {up }}-I_{\text {down }}\right) /\left(I_{\text {up }}+I_{\text {down }}\right)$ is shown.

energy of $8.2 \mathrm{eV}$ at the $\bar{\Gamma}$ point. With increasing of $k_{\|}$the $\pi$ state disperses to Fermi level, and crosses it near the $\bar{K}$ point of BZ of graphene, corresponding to $k_{\|}=1.7 \AA^{-1}$. In the region of the $\bar{K}$ point, the $\pi$ state is characterized by linearlike dispersion. Besides the main branch of the $\pi$ state of graphene, additional replicas of the $\pi$ states are visible. The most pronounced replica marked as " $\mathrm{R}$ " reaches a BE of $0.8 \mathrm{eV}$ at $k_{\|}=1.4 \AA^{-1}$. It has been shown [33,34], that the main branch of the $\pi$ state exhibits minigaps, opened in regions of the intersections with the replicated bands. For the MG/Ir(111) the intersection between the main branch and the replica of the $\pi$ state in the $\bar{\Gamma} \bar{K}$ direction takes place at the $\mathrm{BE}$ of $1 \mathrm{eV}$, and the width of the minigap was established around $0.23 \mathrm{eV}$ [34]. Since graphene on $\operatorname{Ir}(111)$ with an intercalated monolayer of $\mathrm{Pt}$ has the same superstructure as in the MG/Ir(111) system the replicated bands and the minigaps have the same behavior. Indeed, in Figs. 1(c) and 1(d) the minigaps are located at the $\mathrm{BE}$ of $0.7 \mathrm{eV}$ both for the $\mathrm{MG} / \mathrm{Ir}(111)$ and for the MG/Pt/Ir(111) systems.

Besides the graphene-derived features, the electronic states related to the $\operatorname{Ir}(111)$ substrate are also visible in the ARPES spectra of MG/Pt/Ir(111). Several branches of bulk Ir $5 d$ states are pronounced in the region of $0-6 \mathrm{eV}$. Crossing of the spin-polarized Ir $5 d$ branches with the $\pi$ state of graphene in the region of the $\mathrm{BE}$ of $3 \mathrm{eV}$ leads to hybridization and corresponding avoided crossing effect of this state. The same effects were observed for the MG/Ir(111) [14]. The authors showed that such structure results in spin-orbit splitting of the $\pi$ states. Our MG/Pt/Ir(111) system is expected to show a similar spin structure of the $\pi$ state. However, some features in the electronic structure near the $\bar{K}$ point of the graphene BZ are different from that in $\mathrm{MG} / \mathrm{Ir}(111)$ system.

Theoretical calculations of the $9.3 \times 9.3$ superstructure are computationally complex, thus for analysis of the modifications of the system after intercalation of $\mathrm{Pt}$ we calculated the DFT band structure without graphene. In Fig. 2(c) one can see that a Pt monolayer on top of $\operatorname{Ir}(111)$ changes the electronic structure in comparison with clean $\operatorname{Ir}(111)$ (see, for example, Ref. [35]). In the region of $k_{\|}=1.2-2.0 \AA^{-1}$ near the Fermi level the bulk electronic structure of $\operatorname{Ir}(111)$ has a local gap. In Fig. 2(b) this gap is marked by a red dashed line. The surface states of $\operatorname{Ir}(111)$ localize near the lower border of the gap. In Fig. 2(c) these states are seen as thin black lines without symbols, because they are localized on the Pt-free surface of 
the slab. The addition of a monolayer of Pt leads to their mixing with the $5 d$ states of $\mathrm{Pt}$ and shifting to lower BE.

Indeed, in Fig. 2(a) one can clearly see the branches of the interface states, marked as "I" located inside an $\operatorname{Ir}(111)$ energy gap. Theoretical calculations of the band structure of the $\mathrm{Pt} / \mathrm{Ir}(111)$ system in Fig. 2(c) predict the BE of these states of 0.4 and $0.5 \mathrm{eV}$ at the $\bar{K}$ point of BZ of $\operatorname{Ir}(111)$. However, in ARPES spectra of MG/Pt/Ir(111) the "I" state has $\mathrm{BE}$ in the region of $0.3-0.4 \mathrm{eV}$. This shift can be explained by their hybridization with the $\pi$ state of graphene. For proving this statement we calculated the DFT band structure of hypothetical graphene on $\mathrm{Pt} / \operatorname{Ir}(111)$ with $1 \times 1$ structure (with enlarged graphene lattice constant). Results are presented in the Supplemental Material [36] and actually show a shift of the interface states to the lower $\mathrm{BE}$ when graphene is atop $\mathrm{Pt} / \mathrm{Ir}(111)$. Moreover, such a hybridization can lead to a spin-dependent avoided-crossing effect, and corresponding modifications of the spin structure of the $\pi$ state of graphene.

In order to analyze the spin-dependent hybridization effects between the graphene and Pt-Ir interface states we carried out ARPES measurements with spin resolution. In Fig. 3 a set of spin-resolved spectra measured at different emission angles is shown. Far from the BZ edges, at $\mathrm{k}_{\|}=1.3 \AA^{-1}$ the $\pi$ state of graphene reveals a hybridization gap, as a result of the avoided-crossing effect, similar to those in graphene on $\operatorname{Ir}(111)$ [14]. Additionally interface states "I" and replicated band " $R$ " can be distinguished in the spin-resolved spectra at the $\mathrm{BE}$ of 0.6 and $1.1 \mathrm{eV}$, respectively.

In the region of $k_{\|}=1.3-1.5 \AA^{-1}$ the replicated branch reaches the BE of $0.9 \mathrm{eV}$, and with further increasing $k_{\|}$the replica " $R$ " crosses the main branch of the $\pi$ state forming a corresponding minigap. Analyzing the spin-resolved spectra we can rather distinguish the spin polarization of the replicated band in the region before the crossing with the branch of the main graphene $\pi$ states. The branches of the Pt-Ir interface states "I" are located at a BE of $0.3-0.4 \mathrm{eV}$ almost without dispersion. The spin splitting of the main branch of the $\pi$ state in this region is found to be about $20 \mathrm{meV}$.

Near the $\bar{K}$ point of the BZ of graphene the intersection of the state "I" and the graphene $\pi$ state is expected, but due to the hybridization these states do not cross, forming a local gap [see Fig. 4]. As a result of spin-dependent hybridization, the $\pi$ state exhibits a pronounced spin splitting at the Fermi level. The value of the splitting is estimated to be $30 \pm 5 \mathrm{meV}$. It is noteworthy that the value of splitting of the $\pi$ state in the region beyond the crossing with the states "I" does not exceed $20 \mathrm{meV}$. Thus, graphene on $\operatorname{Ir}(111)$ with an intercalated monolayer of $\mathrm{Pt}$ is characterized by spin-orbit splitting of the $\pi$ state, which depends on the position in the BZ. Furthermore, a pronounced spin polarization of the replicated band of the $\pi$ state is observed.

\section{DISCUSSION}

Intercalation of various materials underneath graphene on $\operatorname{Ir}(111)$ may induce many intriguing effects relevant to the electronic and spin structure of graphene. First of all, in Ref. [37] authors showed that intercalation of oxygen leads to decoupling of graphene from the substrate with the shift of the Dirac point by $0.57 \mathrm{eV}$ above the Fermi level.

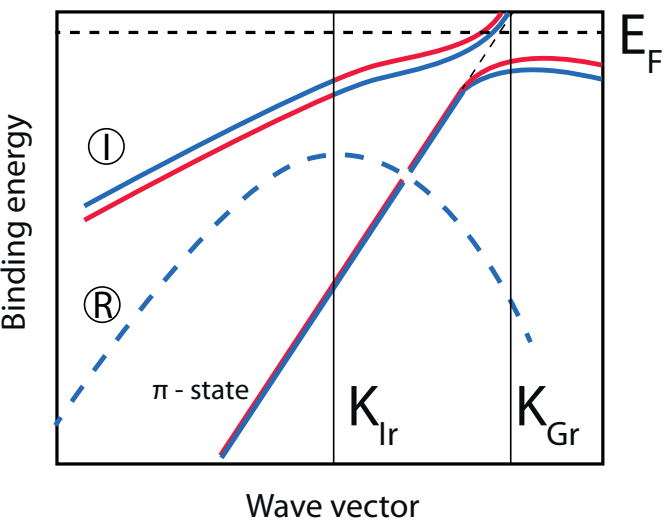

FIG. 4. (Color online) Schematic behavior of the graphene and Pt-Ir interface states near the $\bar{K}$ point of the BZ of graphene.

On the other hand, $\mathrm{Cu}$ intercalated graphene on $\operatorname{Ir}(111)$ is characterized by a gap between the $\pi$ and $\pi *$ states, opened by lifting the sublattice symmetry due to hybridization of the $\mathrm{Cu}$ and graphene bands [38]. At the same time, the result of intercalation of cobalt is the formation of a magnetic moment in the graphene layer [39]. Finally, the most intriguing recent phenomena is a spatially varied SOI and QSH phase in graphene induced by intercalation of $\mathrm{Pb}$ islands [21].

In accordance with a number of works [17-19] a QSH phase in graphene was predicted to be formed due to deposition of certain heavy adatoms on graphene induced by enhanced SO coupling strength. Moreover, it has been predicted that the topological phase can be developed in transition metals (Re [19] and Mn [40]) intercalated graphene with formation of the increased SO gap due to orbital coupling between the graphene $\pi$ states and transition metal $d$ states. For the $\mathrm{Pb}$ intercalated graphene on $\operatorname{Ir}(111)$ [21] the most important factor for formation of the topological phase (and corresponding generation of a pseudomagnetic field resulting in the formation of Landau-like levels) was a spatial gradient of SOI along the surface formed by a $c(4 \times 2)$ superstructure.

It is interesting that for graphene on $\operatorname{Ir}(111)$ itself a formation of the SO gap and corresponding topological properties is not expected despite the formation of a $(9.3 \times 9.3)$ superstructure. In this case hybridization with the $d$ states of $\operatorname{Ir}(111)$ takes place far from the region of the Dirac point, and Rashba SOI (Rashba splitting of the $\pi$ state is around $50 \mathrm{meV}$ ) appears to be higher than "intrinsic" SOI. In the current work we try to modify the relation between Rashba and "intrinsic" SOI in graphene on $\operatorname{Ir}(111)$ by intercalation of a monolayer of Pt.

As we noted before, the main change in the electronic structure of the MG/Ir(111) system after intercalation of a Pt monolayer is the shifting of the spin-orbit split interface states towards the Fermi level. In the region of $k_{\|}$far from the place of the intersection with these states, the $\pi$ state of graphene has a spin splitting of about $20 \mathrm{meV}$. This value does not depend on $k_{\|}$(in the region ascribed above), thus we can attribute it to the Rashba splitting. This decreased value of the spin-orbit splitting can be a result of the difference in the interface potential gradient for MG on $\operatorname{Ir}(111)$ with and without an intercalated $\mathrm{Pt}$ monolayer. Indeed, the position of the Dirac 
point for graphene on $\mathrm{Pt} / \mathrm{Ir}(111)$ (around $0.2 \mathrm{eV}$ above the Fermi level) is similar to that for graphene on bulk $\mathrm{Pt}(111)$ and can be explained by changing of the interface dipole and the corresponding charge transfer between graphene and the substrate relative to the MG/Ir(111). Charge transfer can modulate the asymmetry of the wave functions and, as a consequence, Rashba spin splitting of the states. Similar effects were observed in the Rashba states of topological insulators in Ref. [41].

Meanwhile, spin-dependent hybridization of the interface states "I" with the $\pi$ state near the $\bar{K}$ point of the BZ of graphene leads to an increase of the spin-orbit spiltting of the $\pi$ state up to $30 \mathrm{meV}$. Moreover, since the graphene states mix with the $5 d$ states the mechanism of the spin splitting at the Fermi level is no longer a simple Rashba SOI. Such hybridization may induce the appearance of the spin-orbit gap in the Dirac point. Note, that variation of SOI in MG/Pt/Ir(111) takes place without changes of the $9.3 \times 9.3$ superstructure. Thus, intercalation of Pt underneath graphene on $\operatorname{Ir}(111)$ can change the relation between Rashba and "intrinsic" SOI and may be used for realizing the topological phase in graphene. This mechanism is different from that proposed in Ref. [21], where $\mathrm{Pb}$ atoms increase $\mathrm{SOI}$ in graphene by hybridization with the $p$ states and enhanced potential gradient.

In the end we have to note other intriguing and important problems. The spin-resolved measurements of the replicated $\pi$ states of graphene on $\operatorname{Ir}(111)$ are quite difficult due to localization of the surface states of Ir at the same region as replicas. After intercalation of a monolayer of Pt underneath graphene on $\operatorname{Ir}(111)$ surface states are shifted, and spin polarization of replica "R" become clearly visible. This effect appears as a complex interplay of SOI, superlattice, and photoemission processes and require further investigations.

\section{CONCLUSIONS}

We have presented an experimental and theoretical analysis of the electronic and spin structure of graphene on $\operatorname{Ir}(111)$ with intercalated monolayer of Pt. Modification of the interface gradient of potential leads to a decrease of the Rashba spin splitting of the $\pi$ state in comparison with that for MG/Ir(111). Meanwhile, shifting of the spin-split interface states in the local gap of $\operatorname{Ir}(111)$ leads to a spin-dependent avoided-crossing effect near the Dirac point that results in an increase of the non-Rashba SOI in graphene.

\section{ACKNOWLEDGMENTS}

The work was partially supported by grants of Saint Petersburg State University for scientific investigations (Grants No. 11.38.271.2014, No. 15.61.202.2015 and No. 11.37.634.2013) and Russian Foundation for Basic Research (RFBR) projects (No. 13-02-91327). We acknowledge the financial support of the University of Basque Country UPV/EHU (Grant No. GIC07-IT-756-13), the Departamento de Educacion del Gobierno Vasco, and the Spanish Ministerio de Ciencia e Innovacion (Grant No. FIS2010-19609-C02-01), the Spanish Ministry of Economy and Competitiveness MINECO (Grant No. FIS2013-48286-C2-1-P), and the Tomsk State University Competitiveness Improvement Program. The calculations were performed on the SKIF-Cyberia supercomputer at Tomsk State University (Russia). The authors also acknowledge support from the Russian-German laboratory at BESSY II and the "German-Russian Interdisciplinary Science Center"(G-RISC) program.
[1] Y. A. Bychkov and E. I. Rashba, JETP Lett. 39, 78 (1984).

[2] M. Hoesch, M. Muntwiler, V. N. Petrov, M. Hengsberger, L. Patthey, M. Shi, M. Falub, T. Greber, and J. Osterwalder, Phys. Rev. B 69, 241401 (2004).

[3] S. V. Eremeev, I. A. Nechaev, Y. M. Koroteev, P. M. Echenique, and E. V. Chulkov, Phys. Rev. Lett. 108, 246802 (2012).

[4] A. M. Shikin, A. Varykhalov, G. V. Prudnikova, D. Usachov, V. K. Adamchuk, Y. Yamada, J. D. Riley, and O. Rader, Phys. Rev. Lett. 100, 057601 (2008).

[5] M. Z. Hasan and C. L. Kane, Rev. Mod. Phys. 82, 3045 (2010).

[6] Y. Ando, J. Phys. Soc. Jpn. 82, 102001 (2013).

[7] A. M. Shikin, I. I. Klimovskikh, S. V. Eremeev, A. A. Rybkina, M. V. Rusinova, A. G. Rybkin, E. V. Zhizhin, J. Sanchez-Barriga, A. Varykhalov, I. P. Rusinov, E. V. Chulkov, K. A. Kokh, V. A. Golyashov, V. Kamyshlov, and O. E. Tereshchenko, Phys. Rev. B 89, 125416 (2014).

[8] I. M. Miron, G. Gaudin, S. Auffret, B. Rodmacq, A. Schuhl, S. Pizzini, J. Vogel, and P. Gambardella, Nat. Mater. 9, 230 (2010).

[9] S. D. Bader and S. S. P. Parkin, Ann. Rev. Condens. Matter Phys. 1, 71 (2010).

[10] A. M. Shikin, A. A. Rybkina, A. G. Rybkin, I. I. Klimovskikh, P. N. Skirdkov, K. A. Zvezdin, and A. K. Zvezdin, Appl. Phys. Lett. 105, 042407 (2014).
[11] A. K. Geim and K. S. Novoselov, Nat. Mater. 6, 183 (2007).

[12] N. Tombros, C. Jozsa, M. Popinciuc, H. T. Jonkman, and B. J. van Wees, Nature (London) 448, 571 (2007).

[13] D. Marchenko, A. Varykhalov, M. R. Scholz, G. Bihlmayer, E. I. Rashba, A. G. Rybkin, A. M. Shikin, and O. Rader, Nat. Commun. 3, 1232 (2012).

[14] D. Marchenko, J. Sanchez-Barriga, M. R. Scholz, O. Rader, and A. Varykhalov, Phys. Rev. B 87, 115426 (2013).

[15] D. Usachov, A. Fedorov, M. M. Otrokov, A. Chikina, O. Vilkov, A. Petukhov, A. G. Rybkin, Y. M. Koroteev, E. V. Chulkov, V. K. Adamchuk, A. Grüneis, C. Laubschat, and D. V. Vyalikh, Nano Lett. 15, 2396 (2015).

[16] C. L. Kane and E. J. Mele, Phys. Rev. Lett. 95, 146802 (2005).

[17] C. Weeks, J. Hu, J. Alicea, M. Franz, and R. Wu, Phys. Rev. X 1, 021001 (2011).

[18] J. Hu, J. Alicea, R. Wu, and M. Franz, Phys. Rev. Lett. 109, 266801 (2012).

[19] Y. Li, P. Tang, P. Chen, J. Wu, B. L. Gu, Y. Fang, S. B. Zhang, and W. Duan, Phys. Rev. B 87, 245127 (2013).

[20] I. I. Klimovskikh, S. S. Tsirkin, A. G. Rybkin, A. A. Rybkina, M. V. Filianina, E. V. Zhizhin, E. V. Chulkov, and A. M. Shikin, Phys. Rev. B 90, 235431 (2014). 
[21] F. Calleja, H. Ochoa, M. Garnica, S. Barja, J. J. Navarro, A. Black, M. M. Otrokov, E. V. Chulkov, A. Arnau, A. L. Vazquez de Parga, F. Guinea, and R. Miranda, Nat. Phys. 11, 43 (2015).

[22] P. Lacovig, M. Pozzo, D. Alfe, P. Vilmercati, A. Baraldi, and S. Lizzit, Phys. Rev. Lett. 103, 166101 (2009).

[23] E. Starodub, A. Bostwick, L. Moreschini, S. Nie, F. E. Gabaly, K. F. McCarty, and E. Rotenberg, Phys. Rev. B 83, 125428 (2011).

[24] D. Usachov, A. Fedorov, O. Vilkov, V. K. Adamchuk, L. V. Yashina, L. Bondarenko, A. A. Saranin, A. Gruneis, and D. V. Vyalikh, Phys. Rev. B 86, 155151 (2012).

[25] M. Kralj, I. Pletikosic, M. Petrovic, P. Pervan, M. Milun, A. T. NDiaye, C. Busse, T. Michely, J. Fujii, and I. Vobornik, Phys. Rev. B 84, 075427 (2011).

[26] C. Busse, P. Lazić, R. Djemour, J. Coraux, T. Gerber, N. Atodiresei, V. Caciuc, R. Brako, A. T. N'Diaye, S. Blügel, J. Zegenhagen, and T. Michely, Phys. Rev. Lett. 107, 036101 (2011).

[27] G. Kresse and J. Hafner, Phys. Rev. B 48, 13115 (1993).

[28] G. Kresse and J. Furthmüller, Comput. Mater. Sci. 6, 15 (1996).

[29] P. E. Blöchl, Phys. Rev. B 50, 17953 (1994).

[30] G. Kresse and D. Joubert, Phys. Rev. B 59, 1758 (1999).

[31] John P. Perdew, Kieron Burke, and Matthias Ernzerhof, Phys. Rev. Lett. 77, 3865 (1996).

[32] D. D. Koelling and B. N. Harmon, J. Phys. C 10, 3107 (1977).
[33] I. Pletikosic, M. Kralj, P. Pervan, R. Brako, J. Coraux, A. T. N'Diaye, C. Busse, and T. Michely, Phys. Rev. Lett. 102, 056808 (2009).

[34] J. Sanchez-Barriga, A. Varykhalov, D. Marchenko, M. R. Scholz, and O. Rader, Phys. Rev. B 85, 201413 (2012).

[35] I. Pletikosic, M. Kralj, D. Sokcevic, R. Brako, P. Lazic, and P. Pervan, J. Phys.: Condens. Matter 22, 135006 (2010).

[36] See Supplemental Material at http://link.aps.org/supplemental/ 10.1103/PhysRevB.92.165402 for DFT calculations of graphene on $\mathrm{Pt} / \mathrm{Ir}(111)$ in $1 \times 1$ structure.

[37] R. Larciprete, S. R. Ulstrup, P. Lacovig, M. Dalmiglio, M. Bianchi, F. Mazzola, L. Hornekær, F. Orlando, A. Baraldi, P. Hofmann, and S. Lizzit, ACS Nano 6, 9551 (2012).

[38] H. Vita, S. Bottcher, K. Horn, E. N. Voloshina, R. E. Ovcharenko, T. Kampen, A. Thissen, and Y. S. Dedkov, Sci. Rep. 4, 5704 (2014).

[39] H. Vita, S. Bottcher, P. Leicht, K. Horn, A. B. Shick, and F. Maca, Phys. Rev. B 90, 165432 (2014).

[40] Y. Li, P. Chen, G. Zhou, J. Li, J. Wu, B. L. Gu, S. B. Zhang, and W. Duan, Phys. Rev. Lett. 109, 206802 (2012).

[41] P. D. C. King, R. C. Hatch, M. Bianchi, R. Ovsyannikov, C. Lupulescu, G. Landolt, B. Slomski, J. H. Dil, D. Guan, J. L. Mi, E. D. L. Rienks, J. Fink, A. Lindblad, S. Svensson, S. Bao, G. Balakrishnan, B. B. Iversen, J. Osterwalder, W. Eberhardt, F. Baumberger, and Ph. Hofmann, Phys. Rev. Lett. 107, 096802 (2011). 